\title{
Quartic interaction vertex in the massive integer higher spin field theory in a constant electromagnetic field
}

\author{
I. L. Buchbinder ${ }^{1,2, a}$, V. A. Krykhtin ${ }^{1, b}$ \\ ${ }^{1}$ Department of Theoretical Physics, Tomsk State Pedagogical University, Tomsk 634061, Russia \\ ${ }^{2}$ National Research Tomsk State University, Tomsk 634050, Russia
}

Received: 23 July 2015 / Accepted: 17 September 2015 / Published online: 27 September 2015

(C) The Author(s) 2015. This article is published with open access at Springerlink.com

\begin{abstract}
We consider the massive integer higher spin fields coupled to an external constant electromagnetic field in flat space of arbitrary dimension and find a gauge invariant quartic interaction vertex which is quadratic in a dynamical higher spin field and quadratic in the external field. The construction of the vertex is based on the BRST approach to higher spin field theory where no off-shell constraints on the fields and on the gauge parameters are imposed from the very beginning (unconstrained formulation).
\end{abstract}

\section{Introduction}

The higher spin field theory is an actively developing trend in modern theoretical and mathematical physics (see e.g. the reviews [1-8]). One of the interesting and important problems here is related with the construction of the Lagrangians describing the interactions of the higher spin fields among themselves and with the various backgrounds (see e.g. [9$38]$ and the references therein).

Most of the papers on interacting Lagrangians of the higher spin fields deal with the cubic approximation. In particular, the cubic vertices of higher spin fields have been constructed in $[9,11,13,14,16-18,20,22-27]$, the cubic vertices for higher spin fields coupled to external electromagnetic and gravitational backgrounds have been elaborated in [28-35]. The quartic approximation of the interaction vertices has been studied much less (see e.g. [36-38]).

When considering interactions of higher spin fields with a nontrivial background one faces several difficulties, such as a possibility of superluminal propagation and violation of the number of physical degrees of freedom. The requirement that the superluminal propagation is absent imposes in general certain conditions on the background fields [39-41]

\footnotetext{
a e-mail: joseph@tspu.edu.ru

be-mail: krykhtin@tspu.edu.ru
}

(see also [42] for a recent discussion). Similarly, when turning on nonzero background fields the invariance of the initial system under its gauge transformations can be partially or completely lost and this means in turn that nonphysical polarizations can appear in the spectrum. The requirement of preserving the physical degrees of freedom generically imposes some extra conditions on the background. Therefore to construct a higher spin field theory on some background we should answer the question whether the background under consideration is physically acceptable, i.e., whether it satisfies the constraints imposed by the above mentioned conditions.

In this paper we study a problem of interaction of massive totally symmetric bosonic higher spin fields with constant electromagnetic (EM) background in Minkowski space of an arbitrary dimension $d$. These higher spin fields are described by tensors with an arbitrary number $s$ of totally symmetric tensorial indices. Our main aim is to derive the gauge invariant Lagrangian using the method of BRST construction [4352] in the quadratic approximation in the field strength $F_{\mu \nu}$ of the external field. This method in fact yields a gauge invariant Lagrangian description for massive higher spin fields in extended Fock space and therefore the Lagrangian will contain, apart from the basic fields, some additional fields, such as Stückelberg fields. Some of these fields are eliminated with the help of gauge transformations, some of the others should be eliminated as a result of the equations of motion. Therefore, in order to have a consistent gauge invariant description for massive higher spin fields, one should have enough gauge freedom and have the "correct" equations of motion, which ensure the absence of ghosts. ${ }^{1}$ One can show that the preservation of physical degrees of freedom indeed takes place for

\footnotetext{
1 One way to check this is to perform a complete gauge fixing in the equations of motion and obtain the equations in terms of basic fields. As a result one obtains equations defining the spectrum of the theory and one may check if it is ghost free or not.
} 
the Lagrangian under consideration, provided that the terms containing the strength of the external field are considered as a perturbation.

The paper is organized as follows. Section 2 contains our main results. After a brief reminder of the construction of Lagrangians for free massive bosonic higher spin fields we introduce an interaction with the background electromagnetic fields by deforming the operators which define the BRST charge. The requirement that the deformed operators form a closed algebra fixes the free parameters which are present in the definition of the deformed operators. Then we construct the corresponding BRST charge and derive the gauge invariant Lagrangian describing the interactions with a constant electromagnetic background. In Sect. 3 we derive from our main Lagrangian, obtained in Sect. 2, a family of Lagrangian formulations with different sets of the auxiliary fields by partially or completely gauge fixing. As examples we obtain first the Lagrangian description in terms of the socalled "quartet formulation" $[53,54]$ (see also $[55,56]$ ) and second the Lagrangian formulation obtained in [36]. Actually the expressions for the operators obtained in Sect. 2 from the very beginning are more general compared with earlier work [36], and hence we obtain new interaction vertices for quartic coupling of the higher spin fields to the constant electromagnetic background. The final section contains the conclusions and a discussion.

\section{BRST Lagrangian construction for massive higher spin bosonic fields on electromagnetic background}

Let us briefly summarize the features of the BRST approach for the construction of the gauge invariant bosonic and fermionic higher spin field Lagrangians [43-52]. First one introduces a set of operators that define the spectrum of the theory. ${ }^{2}$ Provided these operators form a closed algebra one builds a nilpotent BRST charge $Q$, which in turn yields a quadratic gauge invariant Lagrangian in terms of the Fock state vectors of the form

$\mathcal{L} \sim\langle\chi|Q| \chi\rangle$

where $|\chi\rangle$ is a vector in an extended Fock space. The gauge invariance of the Lagrangian under the linear gauge transformations

$\delta|\chi\rangle=Q|\Lambda\rangle$

is guaranteed by the nilpotency of the BRST charge, $Q^{2}=0$.

${ }^{2}$ In the free theory the spectrum is given with the help of the relations defining either reducible or irreducible representations of the Poincaré or AdS group.
The situation can be more complicated if the closure of the algebra of the initial set of operators requires inclusion of certain additional operators into the above set. These extra operators can impose too strong conditions on the field $|\chi\rangle$ so that there will be no a nonzero solution to the equation

$Q|\chi\rangle=0$

This problem is overcome as follows [43-52]. One introduces the additional sets of oscillator variables and builds an auxiliary representation of the generators of the algebra (i.e. of the operators under consideration) in terms of these new variables. Then one defines a modified set of operators as a sum of new and old ones and considers the problem in an extended Fock space (including the new oscillators). After that, one builds a BRST charge for the modified generators in the standard way, since the generators form a closed algebra. It allows us to construct a Lagrangian on the base of the BRST charge under consideration.

After this reminder let us turn to a description of massive bosonic higher spin fields. To this end we introduce the Fock space spanned by the oscillators,

$\left[a_{\mu}, a_{v}^{+}\right]=\eta_{\mu \nu}, \quad \eta_{\mu \nu}=\operatorname{diag}(-1,1, \ldots, 1)$,

and consider the operators

$l_{0}^{\prime}=\partial^{2}-m^{2}, \quad l_{1}^{\prime}=i a^{\mu} \partial_{\mu}, \quad l_{2}^{\prime}=\frac{1}{2} a^{\mu} a_{\mu}$.

The first of the operators in (2.5) corresponds to the KleinGordon operator for the massive boson, the second operator is a divergence operator, and the third one is an operator which takes a trace. In order to have a hermitian BRST charge, we also introduce operators which are hermitian conjugate to the operators $l_{1}^{\prime}$ and $l_{2}^{\prime}$ :

$l_{1}^{\prime+}=i a^{+\mu} \partial_{\mu}, \quad l_{2}^{\prime+}=\frac{1}{2} a^{+\mu} a_{\mu}^{+}$.

Finally in order to close the algebra one introduces the extra operators

$g_{0}^{\prime}=a^{+\mu} a_{\mu}+\frac{d}{2}$

and $g_{m}^{\prime}=m^{2}$. The operator $g_{0}^{\prime}$ is a "particle" number operator and its eigenvalues are always strictly positive. Therefore, we have a situation as described earlier in this section. We introduce two sets of additional bosonic oscillator variables with the commutation relations

$\left[b_{1}, b_{1}^{+}\right]=1, \quad\left[b_{2}, b_{2}^{+}\right]=1$.

Using these new variables one can build an auxiliary representation for the original operators and define modified 
operators thus:

$$
\begin{aligned}
& l_{0}=\partial^{2}-m^{2}, \\
& l_{1}=i a^{\mu} \partial_{\mu}+m b_{1} \quad l_{1}^{+}=i a^{+\mu} \partial_{\mu}+m b_{1}^{+}, \\
& l_{2}=\frac{1}{2} a^{\mu} a_{\mu}+\frac{1}{2} b_{1}^{2}+\left(b_{2}^{+} b_{2}+h\right) b_{2} \\
& \quad l_{2}^{+}=\frac{1}{2} a^{+\mu} a_{\mu}^{+}+\frac{1}{2} b_{1}^{+2}+b_{2}^{+}, \\
& g_{0}=a_{\mu}^{+} a^{\mu}+b_{1}^{+} b_{1}+2 b_{2}^{+} b_{2}+\frac{d+1}{2}+h \quad g_{m}=0,
\end{aligned}
$$

where $h$ is an arbitrary real constant. The algebra of these operators is given by Table 1 .

In order to introduce an interaction of the bosonic fields with an external constant EM background field $F_{\mu \nu}=$ const , we shall proceed as follows. First we replace all the partial derivatives by the $U$ (1) covariant ones, $D_{\mu}=\partial_{\mu}-i e A_{\mu}$, and include into the expressions of the operators ${ }^{3}$ (2.9)-(2.12) terms which vanish in the limit $F_{\mu \nu} \rightarrow 0$. After that we require that the new operators form a closed algebra.

Before writing an ansatz for the operators let us note that since the trace of a field and its traceless part are independent from each other one can shift the trace of a field so that the traceless condition remains unchanged. Thus we suppose that the operators related with the traceless condition $l_{2}, l_{2}^{+}$as well as the number operator $g_{0}$ remain unchanged,

$$
L_{2}=l_{2}, \quad L_{2}^{+}=l_{2}^{+} \quad G_{0}=g_{0} .
$$

Moreover, since the oscillator variables $b_{2}, b_{2}^{+}$in (2.8) are included only in the operators (2.13) (see also the expressions (2.11)-(2.12)) we assume that these variables are not present in the expressions of the operators $L_{0}, L_{1}$, and $L_{1}^{+}$.

Since we are going to consider first the approximation linear in $F_{\mu \nu}$ we take the following ansatz for the operators:

$$
\begin{aligned}
L_{1}= & i a^{\alpha} D_{\alpha}+m b_{1}+\frac{i e}{m} a^{+\mu} F_{\mu \alpha} a^{\alpha} \sum_{k=0}^{\infty} \alpha_{0(k)}\left(b_{1}^{+}\right)^{k}\left(b_{1}\right)^{k+1} \\
& +\frac{e}{m^{2}} a^{\alpha} F_{\alpha \sigma} D^{\sigma} \sum_{k=0}^{\infty} \beta_{0(k)}\left(b_{1}^{+}\right)^{k}\left(b_{1}\right)^{k} \\
& +\frac{e}{m^{2}} a^{+\mu} F_{\mu \sigma} D^{\sigma} \sum_{k=0}^{\infty} \gamma_{0(k)}\left(b_{1}^{+}\right)^{k}\left(b_{1}\right)^{k+2}
\end{aligned}
$$

$$
\begin{aligned}
L_{0}= & D^{2}-m^{2}+i e a^{+\mu} F_{\mu \alpha} a^{\alpha} \sum_{k=0}^{\infty} \xi_{0(k)}\left(b_{1}^{+}\right)^{k}\left(b_{1}\right)^{k} \\
& +\frac{e}{m} a^{+\mu} F_{\mu \sigma} D^{\sigma} \sum_{k=0}^{\infty} \zeta_{0(k)}\left(b_{1}^{+}\right)^{k}\left(b_{1}\right)^{k+1}
\end{aligned}
$$

\footnotetext{
3 We shall denote these new operators by the corresponding capital letters.
}

$$
\begin{aligned}
& +\frac{e}{m} a^{\alpha} F_{\alpha \sigma} D^{\sigma} \sum_{k=0}^{\infty} \zeta_{0(k)}^{\prime}\left(b_{1}^{+}\right)^{k+1}\left(b_{1}\right)^{k} \\
L_{1}^{+}= & i a^{+\mu} D_{\mu}+m b_{1}^{+}+\frac{i e}{m} a^{+\mu} F_{\mu \alpha} a^{\alpha} \sum_{k=0}^{\infty} \alpha_{0(k)}^{\prime}\left(b_{1}^{+}\right)^{k+1}\left(b_{1}\right)^{k} \\
& +\frac{e}{m^{2}} a^{+\mu} F_{\mu \sigma} D^{\sigma} \sum_{k=0}^{\infty} \beta_{0(k)}^{\prime}\left(b_{1}^{+}\right)^{k}\left(b_{1}\right)^{k} \\
& +\frac{e}{m^{2}} a^{\alpha} F_{\alpha \sigma} D^{\sigma} \sum_{k=0}^{\infty} \gamma_{0(k)}^{\prime}\left(b_{1}^{+}\right)^{k+2}\left(b_{1}\right)^{k},
\end{aligned}
$$

where $\alpha_{0(k)}, \ldots, \gamma_{0(k)}^{\prime}$ are arbitrary complex constants and the rest of the operators (2.11)-(2.12) are unchanged, as one can see from Eq. (2.13). Let us note that the above relations can be treated as the deformations of the corresponding relations of the free theory by the terms linear in $F_{\mu \nu}$.

Let us point out that the ansatz for the operators $L_{1}, L_{0}$, and $L_{1}^{+}$(2.14)-(2.16) is not the most general one. The ansatz is taken on the basis of the following "minimal" rule. Let us consider the operators (2.14)-(2.16) in the free theory, replace the partial derivatives by the covariant ones, and calculate the commutators. Obviously the algebra will not be closed. Then one adds to these operators the minimal number of terms linear in $F_{\mu \nu}$ in such a way that the algebra is closed in the linear approximation. One can see that according to this "minimal" rule the Lorentz indices of the creation and annihilation operators are always contracted with an index or indices of $F_{\mu \nu}$. In principle, it is possible to consider other deformations of the free theory by the terms linear in $F_{\mu \nu}$. For example, one can add to $L_{1}$ a term of the form $a_{\mu}^{+} a^{\mu} a^{\alpha} F_{\alpha \sigma} D^{\sigma}$, but this term does not obey the "minimal" rule.

From the requirement of the $L_{0}$ to be hermitian, from the condition $\left(L_{1}\right)^{+}=L_{1}^{+}$, and from the requirement that the total system of operators forms a closed algebra in the linear approximation, one finds the expressions for constants which are present in (2.14)-(2.16) and these constants are expressed through four arbitrary real constants $c_{1}, c_{2}, c_{3}$, and $c_{4}$. These expressions are summarized in the Appendix.

Let us consider the second order approximation in $F_{\mu \nu}$. The ansatz for the corrections to the operators $L_{1}, L_{0}$, and $L_{1}^{+}$in this case looks like

$$
\begin{aligned}
L_{1}^{(2)}= & \frac{e^{2}}{m^{3}} F^{2} \sum_{k=0}^{\infty} \alpha_{1 k}\left(b_{1}^{+}\right)^{k}\left(b_{1}\right)^{k+1} \\
& +\frac{e^{2}}{m^{3}} a^{+\mu} F_{\mu \alpha}^{2} a^{\alpha} \sum_{k=0}^{\infty} \alpha_{2 k}\left(b_{1}^{+}\right)^{k}\left(b_{1}\right)^{k+1} \\
& +\frac{e^{2}}{m^{3}}\left(a^{+\mu} F_{\mu \alpha} a^{\alpha}\right)^{2} \sum_{k=0}^{\infty} \alpha_{3 k}\left(b_{1}^{+}\right)^{k}\left(b_{1}\right)^{k+1} \\
& +\frac{e^{2}}{m^{3}} F_{\alpha \beta}^{2} a^{\alpha \beta} \sum_{k=0}^{\infty} \alpha_{4 k}\left(b_{1}^{+}\right)^{k+1}\left(b_{1}\right)^{k}
\end{aligned}
$$




$$
\begin{aligned}
& +\frac{e^{2}}{m^{3}} a^{+\mu \nu} F_{\mu \nu}^{2} \sum_{k=0}^{\infty} \alpha_{5 k}\left(b_{1}^{+}\right)^{k}\left(b_{1}\right)^{k+3} \\
& +\frac{i e^{2}}{m^{4}} a^{\alpha} F_{\alpha \sigma}^{2} D^{\sigma} \sum_{k=0}^{\infty} \beta_{1 k}\left(b_{1}^{+}\right)^{k}\left(b_{1}\right)^{k} \\
& +\frac{i e^{2}}{m^{4}} a^{+\mu} F_{\mu \sigma}^{2} D^{\sigma} \sum_{k=0}^{\infty} \gamma_{1 k}\left(b_{1}^{+}\right)^{k}\left(b_{1}\right)^{k+2} \\
& +\frac{i e^{2}}{m^{4}}\left(a^{+\mu} F_{\mu \alpha} a^{\alpha}\right) a^{\alpha} F_{\alpha \sigma} D^{\sigma} \sum_{k=0}^{\infty} \beta_{2 k}\left(b_{1}^{+}\right)^{k}\left(b_{1}\right)^{k} \\
& +\frac{i e^{2}}{m^{4}} a^{+\mu} F_{\mu \sigma} D^{\sigma}\left(a^{+\mu} F_{\mu \alpha} a^{\alpha}\right) \sum_{k=0}^{\infty} \gamma_{2 k}\left(b_{1}^{+}\right)^{k}\left(b_{1}\right)^{k+2} \text {, } \\
& L_{0}^{(2)}=\frac{e^{2}}{m^{2}} F^{2} \sum_{k=0}^{\infty} \xi_{1 k}\left(b_{1}^{+}\right)^{k}\left(b_{1}\right)^{k} \\
& +\frac{e^{2}}{m^{2}} a^{+\mu} F_{\mu \alpha}^{2} a^{\alpha} \sum_{k=0}^{\infty} \xi_{2 k}\left(b_{1}^{+}\right)^{k}\left(b_{1}\right)^{k} \\
& +\frac{e^{2}}{m^{2}}\left(a^{+\mu} F_{\mu \alpha} a^{\alpha}\right)^{2} \sum_{k=0}^{\infty} \xi_{3 k}\left(b_{1}^{+}\right)^{k}\left(b_{1}\right)^{k} \\
& +\frac{e^{2}}{m^{2}} F_{\alpha \beta}^{2} a^{\alpha \beta} \sum_{k=0}^{\infty} \xi_{4 k}\left(b_{1}^{+}\right)^{k+2}\left(b_{1}\right)^{k} \\
& +\frac{e^{2}}{m^{2}} a^{+\mu \nu} F_{\mu \nu}^{2} \sum_{k=0}^{\infty} \xi_{4 k}^{\prime}\left(b_{1}^{+}\right)^{k}\left(b_{1}\right)^{k+2} \\
& +\frac{i e^{2}}{m^{3}} a^{\alpha} F_{\alpha \sigma}^{2} D^{\sigma} \sum_{k=0}^{\infty} \zeta_{1 k}\left(b_{1}^{+}\right)^{k+1}\left(b_{1}\right)^{k} \\
& +\frac{i e^{2}}{m^{3}} a^{+\mu} F_{\mu \sigma}^{2} D^{\sigma} \sum_{k=0}^{\infty} \zeta_{1 k}^{\prime}\left(b_{1}^{+}\right)^{k}\left(b_{1}\right)^{k+1} \\
& +\frac{i e^{2}}{m^{3}}\left(a^{+\mu} F_{\mu \alpha} a^{\alpha}\right) a^{\alpha} F_{\alpha \sigma} D^{\sigma} \sum_{k=0}^{\infty} \zeta_{2 k}\left(b_{1}^{+}\right)^{k+1}\left(b_{1}\right)^{k} \\
& +\frac{i e^{2}}{m^{3}} a^{+\mu} F_{\mu \sigma} D^{\sigma}\left(a^{+\mu} F_{\mu \alpha} a^{\alpha}\right) \sum_{k=0}^{\infty} \zeta_{2 k}^{\prime}\left(b_{1}^{+}\right)^{k}\left(b_{1}\right)^{k+1} \\
& +\frac{e^{2}}{m^{4}} F_{\sigma \tau}^{2} D^{\sigma} D^{\tau} \sum_{k=0}^{\infty} \zeta_{3 k}\left(b_{1}^{+}\right)^{k}\left(b_{1}\right)^{k} \\
& +\frac{e^{2}}{m^{4}}\left(a^{+\mu} F_{\mu \sigma} D^{\sigma}\right)\left(a^{\alpha} F_{\alpha \sigma} D^{\sigma}\right) \sum_{k=0}^{\infty} \zeta_{4 k}\left(b_{1}^{+}\right)^{k}\left(b_{1}\right)^{k} \\
& +\frac{e^{2}}{m^{4}}\left(a^{\alpha} F_{\alpha \sigma} D^{\sigma}\right)^{2} \sum_{k=0}^{\infty} \zeta_{5 k}\left(b_{1}^{+}\right)^{k+2}\left(b_{1}\right)^{k} \\
& +\frac{e^{2}}{m^{4}}\left(a^{+\mu} F_{\mu \sigma} D^{\sigma}\right)^{2} \sum_{k=0}^{\infty} \zeta_{5 k}^{\prime}\left(b_{1}^{+}\right)^{k}\left(b_{1}\right)^{k+2},
\end{aligned}
$$

$$
\begin{aligned}
L_{1}^{+(2)}= & \frac{e^{2}}{m^{3}} F^{2} \sum_{k=0}^{\infty} \alpha_{1 k}^{\prime}\left(b_{1}^{+}\right)^{k+1}\left(b_{1}\right)^{k} \\
& +\frac{e^{2}}{m^{3}} a^{+\mu} F_{\mu \alpha}^{2} a^{\alpha} \sum_{k=0}^{\infty} \alpha_{2 k}^{\prime}\left(b_{1}^{+}\right)^{k+1}\left(b_{1}\right)^{k} \\
& +\frac{e^{2}}{m^{3}}\left(a^{+\mu} F_{\mu \alpha} a^{\alpha}\right)^{2} \sum_{k=0}^{\infty} \alpha_{3 k}^{\prime}\left(b_{1}^{+}\right)^{k+1}\left(b_{1}\right)^{k} \\
& +\frac{e^{2}}{m^{3}} a^{+\mu \nu} F_{\mu \nu}^{2} \sum_{k=0}^{\infty} \alpha_{4 k}^{\prime}\left(b_{1}^{+}\right)^{k}\left(b_{1}\right)^{k+1} \\
& +\frac{e^{2}}{m^{3}} F_{\alpha \beta}^{2} a^{\alpha \beta} \sum_{k=0}^{\infty} \alpha_{5 k}^{\prime}\left(b_{1}^{+}\right)^{k+3}\left(b_{1}\right)^{k} \\
& +\frac{i e^{2}}{m^{4}} a^{+\mu} F_{\mu \sigma}^{2} D^{\sigma} \sum_{k=0}^{\infty} \beta_{1 k}^{\prime}\left(b_{1}^{+}\right)^{k}\left(b_{1}\right)^{k} \\
& +\frac{i e^{2}}{m^{4}} a^{\alpha} F_{\alpha \sigma}^{2} D^{\sigma} \sum_{k=0}^{\infty} \gamma_{1 k}^{\prime}\left(b_{1}^{+}\right)^{k+2}\left(b_{1}\right)^{k} \\
& +\frac{i e^{2}}{m^{4}} a^{+v} F_{\nu \sigma} D^{\sigma}\left(a^{+\mu} F_{\mu \alpha} a^{\alpha}\right) \sum_{k=0}^{\infty} \beta_{2 k}^{\prime}\left(b_{1}^{+}\right)^{k}\left(b_{1}\right)^{k} \\
&
\end{aligned}
$$

where $\alpha_{i(k)}, \ldots, \gamma_{j(k)}^{\prime}$ are arbitrary complex constants.

Analogously to the linear approximation from the requirement of the $L_{0}$ to be hermitian, from the condition $\left(L_{1}\right)^{+}=$ $L_{1}^{+}$, and from the requirement that the total system of operators forms a closed algebra in the quadratic approximation, one finds the expressions for the constants which are present in (2.17)-(2.19). These expressions are summarized in the appendix and all of them are expressed through eight independent real constants. One should note that the quadratic approximation gives additional restrictions on the arbitrary real constants $c_{1}$ and $c_{3}$ of the linear approximation (B.1).

Note that a similar problem was considered in [36], but our approach leads to a more general Lagrangian construction. We found two more arbitrary constants in the linear approximation and six ${ }^{4}$ more arbitrary constants in the quadratic approximation because, unlike [36], we do not require from the very beginning that the coefficients in (2.14)-(2.16) and (2.17)-(2.19) must satisfy reality conditions. As one can see from the appendix, complex coefficients are also acceptable.

The new operators form an algebra which is the same as in the free theory and it is given in Table 1.

After we have achieved the closure of the algebra for the operators, the next step is to construct the corresponding BRST charge. This procedure follows closely the one developed for the bosonic fields in [43-52] to which we refer for more details. First we construct the standard BRST operator

\footnotetext{
4 Two of the four arbitrary constants in the quadratic approximation in [36] are related with the deformation of the trace condition.
} 
Table 1 The algebra of the operators

\begin{tabular}{lllllll}
\hline$\downarrow \downarrow, \rightarrow\}$ & $L_{0}$ & $L_{1}$ & $L_{1}^{+}$ & $L_{2}$ & $L_{2}^{+}$ & $G_{0}$ \\
\hline$L_{0}$ & 0 & 0 & 0 & 0 & 0 & 0 \\
$L_{1}$ & 0 & 0 & $-L_{0}$ & 0 & $L_{1}^{+}$ & $L_{1}$ \\
$L_{1}^{+}$ & 0 & $L_{0}$ & 0 & $-L_{1}$ & 0 & $-L_{1}^{+}$ \\
$L_{2}$ & 0 & 0 & $L_{1}$ & 0 & $G_{0}$ & $2 L_{2}$ \\
$L_{2}^{+}$ & 0 & $-L_{1}^{+}$ & 0 & $-G_{0}$ & 0 & $-2 L_{2}^{+}$ \\
$G_{0}$ & 0 & $-L_{1}$ & $L_{1}^{+}$ & $-2 L_{2}$ & $2 L_{2}^{+}$ & 0 \\
\hline
\end{tabular}

on the basis of the operators (2.13)-(2.19),

$$
\begin{aligned}
Q= & \eta_{0} L_{0}+\eta_{1}^{+} L_{1}+\eta_{1} L_{1}^{+}+\eta_{2}^{+} L_{2}+\eta_{2} L_{2}^{+}+\eta_{G} G_{0} \\
& +\eta_{1}^{+} \eta_{1} \mathcal{P}_{0}-\eta_{2}^{+} \eta_{2} \mathcal{P}_{G}+\left(\eta_{G} \eta_{1}^{+}-\eta_{2}^{+} \eta_{1}\right) \mathcal{P}_{1} \\
& +\left(\eta_{1} \eta_{G}-\eta_{1}^{+} \eta_{2}\right) \mathcal{P}_{1}^{+} \\
& +2 \eta_{G} \eta_{2}^{+} \mathcal{P}_{2}+2 \eta_{2} \eta_{G} \mathcal{P}_{2}^{+} .
\end{aligned}
$$

Here $\eta_{0}, \eta_{1}^{+}, \eta_{1}, \eta_{2}^{+}, \eta_{2}$, and $\eta_{G}$ are fermionic ghost "coordinates" corresponding to their canonically conjugate ghost "momenta" $\mathcal{P}_{0}, \mathcal{P}_{1}, \mathcal{P}_{1}^{+}, \mathcal{P}_{2}, \mathcal{P}_{2}^{+}$, and $\mathcal{P}_{G}$. They obey the anticommutation relations

$$
\begin{aligned}
& \left\{\eta_{1}, \mathcal{P}_{1}^{+}\right\}=\left\{\mathcal{P}_{1}, \eta_{1}^{+}\right\}=\left\{\eta_{2}, \mathcal{P}_{2}^{+}\right\}=\left\{\mathcal{P}_{2}, \eta_{2}^{+}\right\} \\
& \quad=\left\{\eta_{0}, \mathcal{P}_{0}\right\}=\left\{\eta_{G}, \mathcal{P}_{G}\right\}=1
\end{aligned}
$$

and possess the standard ghost number distribution, $g h(\eta)=$ $-g h(\mathcal{P})=1$, which gives $g h(Q)=1$.

For the subsequent computations it is convenient to represent the BRST operator (2.20) in the form

$$
\begin{aligned}
Q= & \tilde{Q}+\eta_{G}\left(N+\frac{d-5}{2}+h\right)+\left(2 q_{1}^{+} q_{1}-\eta_{2}^{+} \eta_{2}\right) \mathcal{P}_{G}, \\
N= & a_{\mu}^{+} a^{\mu}+b_{1}^{+} b_{1}+2 b_{2}^{+} b_{2}+\eta_{1}^{+} \mathcal{P}_{1}+\mathcal{P}_{1}^{+} \eta_{1} \\
& +2 \eta_{2}^{+} \mathcal{P}_{2}+2 \mathcal{P}_{2}^{+} \eta_{2}, \\
\tilde{Q}= & \eta_{0} L_{0}+\Delta Q+\eta_{1}^{+} \eta_{1} \mathcal{P}_{0}, \\
\Delta Q= & \eta_{1}^{+} L_{1}+\eta_{1} L_{1}^{+}+\eta_{2}^{+} L_{2}+\eta_{2} L_{2}^{+} \\
& \quad-\eta_{2}^{+} \eta_{1} \mathcal{P}_{1}-\eta_{1}^{+} \eta_{2} \mathcal{P}_{1}^{+} .
\end{aligned}
$$

Next we choose the following representation for the vacuum in the Fock space:

$$
\left(\mathcal{P}_{0}, \mathcal{P}_{G}, \eta_{1}, \mathcal{P}_{1}, \eta_{2}, \mathcal{P}_{2}\right)|0\rangle=0,
$$

and we suppose that the vectors and gauge parameters do not depend on $\eta_{G}$,

$$
\begin{aligned}
|\chi\rangle= & \sum_{k_{i}}\left(\eta_{0}\right)^{k_{1}}\left(\eta_{1}^{+}\right)^{k_{2}}\left(\mathcal{P}_{1}^{+}\right)^{k_{3}}\left(\eta_{2}^{+}\right)^{k_{4}}\left(\mathcal{P}_{2}^{+}\right)^{k_{5}}\left(b_{1}^{+}\right)^{k_{6}}\left(b_{2}^{+}\right)^{k_{7}} a^{+\mu_{1}} \\
& \cdots a^{+\mu_{k_{0}}} \chi_{\mu_{1} \cdots \mu_{k_{0}}}^{k_{1} \cdots k_{7}}(x)|0\rangle .
\end{aligned}
$$

The sum in (2.23) is taken over $k_{0}, k_{6}, k_{7}$, running from 0 to infinity, and over $k_{1}, k_{2}, k_{3}, k_{4}, k_{5}$, running from 0 to 1 .
Then we derive from Eqs. (2.3) as well as from the reducible gauge transformations, Eq. (2.2), a sequence of relations

$$
\begin{array}{llr}
\tilde{Q}|\chi\rangle=0, & \left(N+\frac{d-5}{2}+h\right)|\chi\rangle=0, & (\epsilon, g h)(|\chi\rangle)=(1,0), \\
\delta|\chi\rangle=\tilde{Q}|\Lambda\rangle, & \left(N+\frac{d-5}{2}+h\right)|\Lambda\rangle=0, & (\epsilon, g h)(|\Lambda\rangle)=(0,-1), \\
(2.25) \\
\delta|\Lambda\rangle=\tilde{Q}\left|\Lambda^{(1)}\right\rangle, & \left(N+\frac{d-5}{2}+h\right)\left|\Lambda^{(1)}\right\rangle=0, & (\epsilon, g h)\left(\left|\Lambda^{(1)}\right\rangle\right)=(1,-2) .
\end{array}
$$

Here $\epsilon$ defines the Grassmann parity of the corresponding fields and parameters of the gauge transformations by $(-1)^{\epsilon}$. The middle equation in (2.24) is a constraint on the possible values of $h$,

$h=-s-\frac{d-5}{2}$.

By fixing the value of the spin, we also fix the parameter $h$, according to (2.27). Having fixed a value of $h$, we then substitute it into each of the expressions (2.24)-(2.26).

It is straightforward to check that Eqs. (2.24) can be obtained from the following Lagrangian [43-52]:

$$
\begin{aligned}
\mathcal{L}= & \int d \eta_{0}\left\langle\chi\left|K_{h} \tilde{Q}\right| \chi\right\rangle \\
= & \left\langle S\left|K_{h} L_{0}\right| S\right\rangle-\left\langle S\left|K_{h} \Delta Q\right| A\right\rangle \\
& -\left\langle A\left|K_{h} \Delta Q\right| S\right\rangle-\left\langle A\left|K_{h} \eta_{1}^{+} \eta_{1}\right| A\right\rangle,
\end{aligned}
$$

where we have decomposed the state vector $|\chi\rangle$ as follows:

$|\chi\rangle=|S\rangle+\eta_{0}|A\rangle$.

In (2.28) the operator $K_{h}$,

$$
\begin{aligned}
& K_{h}=\sum_{n=0}^{\infty}|n\rangle \frac{C(n, h)}{n !}\langle n|, \quad| n\rangle=\left(b_{2}^{+}\right)^{n}|0\rangle, \quad h+s+\frac{d-5}{2}=0, \\
& C(n, h)=h(h+1)(h+2) \ldots(h+n-1), \quad C(0, h)=1,
\end{aligned}
$$

is needed to maintain Hermiticity of the Lagrangian since as one can see from the auxiliary representations for the operators (2.11) one has $\left(l_{2}\right)^{+} \neq l_{2}^{+}$.

The Lagrangian (2.28) describes the interaction of massive bosonic fields with constant electromagnetic field in a quadratic approximation in $F_{\mu \nu}$ and it is our main result. It contains, apart from the basic field $\varphi_{\mu_{1} \ldots \mu_{s}}(x)$ in $|S\rangle$ (2.29),

$|S\rangle=\varphi_{\mu_{1} \ldots \mu_{n}}(x) a^{+\mu_{1}} \ldots a^{+\mu_{n}}|0\rangle+\cdots$,

a number of additional fields, ${ }^{5}$ whose number increases with the spin value.

In the next section we show that after partially or completely gauge fixing one can obtain different Lagrangian formulations with a smaller number of additional fields.

\footnotetext{
5 In the decomposition (2.23) they are coefficients in the summands which contain at least one creation operator different from $a^{+\mu}$.
} 


\section{Partial gauge fixing and different Lagrangian formulations}

In this section we are going to obtain from (2.28) the various equivalent Lagrangian formulations by partially fixing the gauge invariance.

First we derive a quartet Lagrangian formulation [53,54]. Initially this formulation was developed for the massless higher spin fields in a flat and AdS background in [53]. Its bosonic version contains six unconstrained fields (one physical field and five additional fields, two of which are Lagrangian multipliers) and one unconstrained gauge parameter. ${ }^{6}$ Using dimensional reduction one can obtain in principle the quartet formulation for massive higher spin fields [54].

To derive the unconstrained formulation from the Lagrangian (2.28) we partially fix the gauge invariance as was done in [43-52] (putting there the curvature to zero, $R=0$ ), except that we will not fix gauge invariance corresponding to the gauge parameter $|\varepsilon\rangle$ (we remove only the dependence on $b_{2}^{+}$),

$$
\begin{aligned}
& |\Lambda\rangle=\mathcal{P}_{1}^{+}|\varepsilon\rangle+\cdots \\
& |\varepsilon\rangle=\sum_{k=0}^{s-1} \frac{1}{k !}\left(b_{1}^{+}\right)^{k}\left|\varepsilon_{n-k-1}\right\rangle \\
& \left|\varepsilon_{s-k-1}\right\rangle=\frac{1}{(s-k) !} a^{+\mu_{1}} \ldots a^{+\mu_{s-k-1}} \varepsilon_{\mu_{1} \ldots \mu_{s-k-1}}(x)|0\rangle .
\end{aligned}
$$

Next one can show that after the gauge fixing some of the remaining fields can be removed with the help of the equations of motion analogously to [53] and the non-vanishing fields in the quartet formulation are

$$
\begin{aligned}
& |S\rangle=\left|S_{1}\right\rangle+\eta_{1}^{+} \mathcal{P}_{1}^{+}\left|S_{2}\right\rangle+\eta_{2}^{+} \mathcal{P}_{1}^{+}\left|S_{4}\right\rangle, \\
& |A\rangle=\mathcal{P}_{1}^{+}\left|A_{1}\right\rangle+\mathcal{P}_{2}^{+}\left|A_{2}\right\rangle+\eta_{1}^{+} \mathcal{P}_{1}^{+} \mathcal{P}_{2}^{+}\left|A_{3}\right\rangle .
\end{aligned}
$$

The Lagrangian and the gauge transformation for the massive bosonic higher spin field interacting with constant electromagnetic field in the quartet formulation are ${ }^{7}$

$$
\begin{aligned}
\mathcal{L}= & \left\langles _ { 1 } \left|\left\{L_{0}\left|s_{1}\right\rangle-L_{1}^{+}\left|a_{1}\right\rangle-L_{2}^{+\prime}\left|a_{2}\right\rangle\right\}-\left\langle s_{2}\right|\left\{L_{0}\left|s_{2}\right\rangle\right.\right.\right. \\
& \left.-L_{1}\left|a_{1}\right\rangle+\left|a_{2}\right\rangle-L_{2}^{+\prime}\left|a_{3}\right\rangle\right\}+\left\langles _ { 4 } \left|\left\{L_{1}\left|a_{2}\right\rangle-L_{1}^{+}\left|a_{3}\right\rangle\right\}\right.\right. \\
& -\left\langlea _ { 1 } \left|\left\{L_{1}\left|s_{1}\right\rangle-L_{1}^{+}\left|s_{2}\right\rangle-\left|a_{1}\right\rangle\right\}-\left\langle a_{2}\right|\left\{L_{2}^{\prime}\left|s_{1}\right\rangle\right.\right.\right. \\
& \left.+\left|s_{2}\right\rangle-L_{1}^{+}\left|s_{4}\right\rangle\right\}+\left\langlea _ { 3 } \left|\left\{L_{2}^{\prime}\left|s_{2}\right\rangle-L_{1}\left|s_{4}\right\rangle\right\}\right.\right.
\end{aligned}
$$

\footnotetext{
${ }^{6}$ Another similar formulation (the so-called triplet formulation) of bosonic fields on Minkowski and $A d S_{d}$ backgrounds contains one physical and two additional fields [57-62] (see also [64] for a recent discussion) and this corresponds to a description of reducible representations of the Poincaré or $S O(d-1,2)$ groups.

7 In order to obtain the triplet formulation [57-63] one should discard the field $\left|s_{4}\right\rangle$ and the Lagrangian multipliers $\left|a_{2}\right\rangle,\left|a_{3}\right\rangle$ in (3.5).
}

$\delta\left|s_{1}\right\rangle=L_{1}^{+}|\varepsilon\rangle, \quad \delta\left|s_{2}\right\rangle=L_{1}|\varepsilon\rangle, \quad \delta\left|s_{4}\right\rangle=L_{2}^{\prime}|\varepsilon\rangle$.

In the above relations the lower-case letters in the state vectors $\left|s_{i}\right\rangle$ and $\left|a_{i}\right\rangle$ denote the parts of the corresponding vectors $\left|S_{i}\right\rangle$ and $\left|A_{i}\right\rangle$ depending only on the oscillators $\left(a_{\mu}, b_{1}\right)$.

Also the operators $L_{2}^{\prime}$ and $L_{2}^{+\prime}$ are the $\left(a_{\mu}, b_{1}\right)$ parts of the operators $L_{2}$ and $L_{2}^{+}(2.11)$,

$L_{2}^{\prime}=\frac{1}{2} a^{\mu} a_{\mu}+\frac{1}{2} b_{1}^{2}, \quad L_{2}^{+\prime}=\frac{1}{2} a^{+\mu} a_{\mu}^{+}+\frac{1}{2} b_{1}^{+2}$.

Next we will show that the Lagrangian formulation, obtained in [36], is a particular case of our general result (2.28). To get such Lagrangian formulations from (3.5) we first partly fix the gauge, removing the field $\left|s_{4}\right\rangle$ with the help of gauge transformations (3.6), and then integrate out all the fields except the field $\left|s_{1}\right\rangle$. The result is

$$
\begin{aligned}
& \mathcal{L}=\left\langle s_{1}\right|\left\{L_{0}-2 L_{2}^{+\prime} L_{0} L_{2}^{\prime}-L_{1}^{+} L_{1}-L_{1}^{+} L_{1}^{+} L_{2}^{\prime}\right. \\
& \left.\quad-L_{2}^{+\prime} L_{1} L_{1}-L_{2}^{+\prime} L_{1}^{+} L_{1} L_{2}^{\prime}\right\}\left|s_{1}\right\rangle \\
& \delta\left|s_{1}\right\rangle=L_{1}^{+}\left|\lambda_{1}\right\rangle
\end{aligned}
$$

where the state $\left|s_{1}\right\rangle$ and the parameter of the gauge transformations $|\varepsilon\rangle$ obey the constraints

$L_{2}^{\prime} L_{2}^{\prime}\left|s_{1}\right\rangle=0, \quad L_{2}^{\prime}|\varepsilon\rangle=0$

Such a partial form of the Lagrangian was obtained in [36], but with less general ${ }^{8}$ expressions for the operators (2.13) (2.16).

We can proceed to obtain the different forms of the Lagrangian formulation possessing the interesting properties. For example, we can resolve constraints on the field and the gauge parameter (3.10). Using the decomposition (3.2) for $|\varepsilon\rangle$ and an analogous decomposition for $\left|s_{1}\right\rangle$

$$
\begin{aligned}
\left|s_{1}\right\rangle= & \sum_{k=0}^{s} \frac{1}{k !}\left(b_{1}^{+}\right)^{k}\left|\varphi_{s-k}\right\rangle \\
& \left|\varphi_{k}\right\rangle \propto \varphi_{\mu_{1} \cdots \mu_{k}}(x) a^{\mu_{1}+} \ldots a^{\mu_{k}+}|0\rangle,
\end{aligned}
$$

we find that the gauge parameters $\left|\varepsilon_{s-1}\right\rangle$ and $\left|\varepsilon_{s-2}\right\rangle$ are not restricted and the other parameters $\left|\epsilon_{k}\right\rangle$ are expressed in terms of their traces,

$$
\left|\varepsilon_{s-2 k-1}\right\rangle=\left(2 l_{2}^{\prime}\right)^{k}\left|\varepsilon_{s-1}\right\rangle, \quad\left|\varepsilon_{s-2 k-2}\right\rangle=\left(2 l_{2}^{\prime}\right)^{k}\left|\varepsilon_{s-2}\right\rangle,
$$

where $l_{2}^{\prime}$ was defined in (2.5). So we may make a gauge transformation using the unrestricted gauge parameters $\left|\varepsilon_{s-1}\right\rangle$ and $\left|\varepsilon_{s-2}\right\rangle$.

\footnotetext{
8 It should be noted that in [36] a deformation was considered of the operators corresponding to the traceless conditions as well. But this deformation is proportional to an arbitrary constant and as we said at the beginning of our paper it can be removed by a field redefinition.
} 
One can do the same for the field $\left|s_{1}\right\rangle$. Due to the restriction (3.10) there are only four independent fields, $\left|\varphi_{s}\right\rangle,\left|\varphi_{s-1}\right\rangle$, $\left|\varphi_{s-2}\right\rangle$, and $\left|\varphi_{s-3}\right\rangle$; all the other fields are expressed through these four fields,

$$
\begin{aligned}
& \left|\varphi_{s-2 k}\right\rangle=k\left(2 l_{2}^{\prime}\right)^{k-1}\left|\varphi_{s-2}\right\rangle-(k-1)\left(2 l_{2}^{\prime}\right)^{k}\left|\varphi_{s}\right\rangle, \\
& \left|\varphi_{s-2 k-1}\right\rangle=k\left(2 l_{2}^{\prime}\right)^{k-1}\left|\varphi_{s-3}\right\rangle-(k-1)\left(2 l_{2}^{\prime}\right)^{k}\left|\varphi_{s-1}\right\rangle .
\end{aligned}
$$

Thus one can obtain ${ }^{9}$ the gauge invariant Lagrangian formulations for a massive bosonic field interacting with a constant electromagnetic field with the help of four fields $\left|\varphi_{s}\right\rangle$, $\left|\varphi_{s-1}\right\rangle,\left|\varphi_{s-2}\right\rangle,\left|\varphi_{s-3}\right\rangle$, and two gauge parameters $\left|\varepsilon_{s-1}\right\rangle$ and $\left|\varepsilon_{s-2}\right\rangle$.

Finally, using the remaining unrestricted gauge parameters $\left|\varepsilon_{s-1}\right\rangle$ and $\left|\varepsilon_{s-2}\right\rangle$ one can remove the fields $\left|\varphi_{s-1}\right\rangle$ and $\left|\varphi_{s-2}\right\rangle$ using a gauge transformation and obtain the Lagrangian formulation in terms of two traceful unrestricted fields: a field $\left|\varphi_{s}\right\rangle$ whose traceless part is a physical field and an additional $\left|\varphi_{s-3}\right\rangle$ field. This Lagrangian has no gauge invariance, since we have already used the entire gauge freedom. It should be noted that if we decompose the traceful fields $\left|\varphi_{s}\right\rangle$ and $\left|\varphi_{s-3}\right\rangle$ in a series of traceless fields we obtain a set of fields which coincides with the set of fields of Singh and Hagen [65].

\section{Conclusions}

In the present paper we have developed the BRST approach to Lagrangian description of massive higher spin bosonic fields coupled to a constant external electromagnetic field and constructed the corresponding quartic interaction vertex. Such a vertex is quadratic in the dynamical higher spin fields and quadratic in the external field. To this end, we deformed the operators underlying the BRST charge, which correspond to the noninteracting bosonic massive higher spin fields, by the terms depending on the electromagnetic field. Our main result is the Lagrangian (2.28), which contains, apart from the basic field, also a number of auxiliary fields which provide the gauge invariant description for massive theory, and the number of these fields grows with the value of the spin.

We have shown that one can partially or completely fix the gauge invariance and obtain from the general Lagrangian (2.28) a family of different Lagrangian formulations with a smaller number of auxiliary fields. As an example we have derived a Lagrangian formulation for the massive bosonic higher spin fields coupled to a constant electromagnetic background in the quartet (unconstrained) formulation (3.5) [53,54] and we obtained the results of Ref. [36] (3.8) as a particular case of the general Lagrangian (2.28).

\footnotetext{
9 Since the Lagrangian formulation is very large, we do not present it here.
}

Since in our previous paper [66] we have considered fermionic higher spin fields it would be naturally interesting to generalize the present results for the case of supersymmetric systems ${ }^{10}$ as well as to consider higher order interactions. The inclusion of a nontrivial gravitational background is another interesting problem to consider (see for example [68-70] for recent progress in these directions). It would be interesting also to establish more connections of the BRST approach with the recent studies in conformal higher spin fields (see for example [71-75]). We hope to address these questions in future publications.

Acknowledgments I.L.B. would like to acknowledge the Mainz Institute for Theoretical Physics (MITP) for enabling to complete some portion of this work. I.L.B. is grateful to the grant for LRSS, project 88.2014.2 and RFBR grants, projects No. 13-02-90430 and No. 15-0203594 for partial support. The research of V.A.K. was also supported in part by the Russian Ministry of Education and Science under contract 3.867.2014/K.

Open Access This article is distributed under the terms of the Creative Commons Attribution 4.0 International License (http://creativecomm ons.org/licenses/by/4.0/), which permits unrestricted use, distribution, and reproduction in any medium, provided you give appropriate credit to the original author(s) and the source, provide a link to the Creative Commons license, and indicate if changes were made.

Funded by $\mathrm{SCOAP}^{3}$.

\section{A The arbitrary parameters in the first order in $F_{\mu \nu}$}

Below we give the expressions for free parameters which are present in Eqs. (2.14)-(2.16)

$$
\begin{aligned}
& \alpha_{0(0)}=c_{1}+i c_{2} \quad \alpha_{0(0)}^{\prime}=c_{1}-i c_{2}, \\
& \alpha_{0(k \geq 1)}=-\alpha_{0(k \geq 1)}^{\prime}=\frac{(-2)^{k}}{k !} i c_{2}, \\
& \beta_{0(0)}=c_{3}+i c_{4} \quad \beta_{0(0)}^{\prime}=-c_{3}+i c_{4}, \\
& \beta_{0(1)}=3-3 c_{1}-4 c_{3}+i c_{2}, \\
& \beta_{0(1)}^{\prime}=-3+3 c_{1}+4 c_{3}+i c_{2}, \\
& \beta_{0(k \geq 2)}=\frac{(-2)^{k-1}}{k !}\left[(k+1)\left(1-c_{1}-2 c_{3}\right)+i c_{2}\right], \\
& \beta_{0(k \geq 2)}^{\prime}=\frac{(-2)^{k-1}}{k !}\left[-(k+1)\left(1-c_{1}-2 c_{3}\right)+i c_{2}\right], \\
& \gamma_{0(0)}=-2 c_{3} \quad \gamma_{0(0)}^{\prime}=2 c_{3}, \\
& \gamma_{0(k \geq 1)}=-\gamma_{0(k \geq 1)}^{\prime}=\frac{(-2)^{k}}{k !}\left(1-c_{1}-2 c_{3}\right), \\
& \xi_{0(0)}=1-2 c_{1}, \\
& \xi_{0(k \geq 1)}=0, \\
& \zeta_{0(0)}=3-2 c_{1}, \quad \zeta_{0(0)}^{\prime}=-3+2 c_{1}, \\
& \zeta_{0((k \geq 1)}=\zeta_{0((k \geq 1)}^{\prime}=0 .
\end{aligned}
$$

Here $c_{1}, c_{2}, c_{3}$, and $c_{4}$ are arbitrary dimensionless constants.

\footnotetext{
10 The Lagrangian formulation of free supersymmetric massive higher spin theory was given in [67].
} 


\section{B The arbitrary parameters in the second order in $\boldsymbol{F}_{\mu \nu}$}

Below we give the expressions for free parameters which are present in Eqs. (2.17)-(2.19)

$\xi_{1(0)}=a_{0}, \quad \xi_{1(1)}=\frac{1}{2}\left(3-2 c_{1}\right), \quad \xi_{1(k \geq 2)}=0$,

$\xi_{2(0)}=\frac{1}{2}\left(3-2 c_{1}\right), \quad \xi_{2(1)}=3-2 c_{1}, \quad \xi_{2(k \geq 2)}=0$,

$\xi_{3(0)}=\frac{11}{4}-7 c_{1}+4 c_{1}^{2}-4 c_{3}+4 c_{1} c_{3}+4 c_{3}^{2}, \quad \xi_{3(k \geq 1)}=0$,

$\xi_{4(0)}=\xi_{4(0)}^{\prime}=-\frac{1}{2}\left(3-2 c_{1}\right), \quad \xi_{4(k \geq 1)}=\xi_{4(k \geq 1)}^{\prime}=0$,

$\zeta_{1(0)}=-4 c_{1}^{2}-6 c_{3} c_{1}+8 c_{1}-4 c_{3}^{2}+7 c_{3}-\frac{17}{4}+3 i c_{4}-2 i c_{1} c_{4}$,

$\zeta_{1(0)}^{\prime}=-4 c_{1}^{2}-6 c_{3} c_{1}+8 c_{1}-4 c_{3}^{2}+7 c_{3}-\frac{17}{4}+2 i c_{4} c_{1}-3 i c_{4}$,

$\zeta_{1(k \geq 1)}=\frac{(-2)^{k-1}}{k !}\left(3-2 c_{1}\right)\left(1-c_{1}-2 c_{3}+i c_{2}\right)$,

$\zeta_{1(k \geq 1)}^{\prime}=\frac{(-2)^{k-1}}{k !}\left(3-2 c_{1}\right)\left(1-c_{1}-2 c_{3}-i c_{2}\right)$,

$\zeta_{2(0)}=6 c_{1}^{2}+8 c_{3} c_{1}-11 c_{1}+8 c_{3}^{2}-8 c_{3}+\frac{11}{2}-2 i c_{2} c_{1}+3 i c_{2}$,

$\zeta_{2(0)}^{\prime}=-6 c_{1}^{2}-8 c_{3} c_{1}+11 c_{1}-8 c_{3}^{2}+8 c_{3}-\frac{11}{2}-2 i c_{2} c_{1}+3 i c_{2}$,

$\zeta_{2(k \geq 1)}=\zeta_{2(k \geq 1)}^{\prime}=i \frac{(-2)^{k}}{k !}\left(3-2 c_{1}\right) c_{2}$,

$\zeta_{3(0)}=-c_{3}^{2}-c_{4}^{2}+2 b_{0}, \quad \zeta_{3(1)}=-4 c_{1}^{2}-8 c_{3} c_{1}+8 c_{1}-4 c_{3}^{2}$,

$$
+10 c_{3}-\frac{17}{4}
$$

$\zeta_{3(k \geq 2)}=-\frac{(-2)^{k-1}}{(k-1) !}\left(3-2 c_{1}\right)\left(1-c_{1}-2 c_{3}\right)$,

$\zeta_{4(0)}=4 c_{3}^{2}+2 c_{3}+2 c_{1}-\frac{7}{4}, \quad \zeta_{4(1)}=12 c_{1}^{2}+24 c_{3} c_{1}-26 c_{1}$,

$$
+8 c_{3}^{2}-32 c_{3}+\frac{29}{2} \text {, }
$$

$\zeta_{4(k \geq 2)}=-\frac{(-2)^{k}}{k !}(k+1)\left(3-2 c_{1}\right)\left(1-c_{1}-2 c_{3}\right)$,

$\zeta_{5(0)}=\zeta_{5(0)}^{\prime}=-\zeta_{4(0)}=\frac{7}{4}-4 c_{3}^{2}-2 c_{3}-2 c_{1}$,

$\zeta_{5(k \geq 1)}=\zeta_{5(k \geq 1)}^{\prime}=\frac{(-2)^{k}}{k !}\left(3-2 c_{1}\right)\left(1-c_{1}-2 c_{3}\right)$,

$\alpha_{1(0)}=-\frac{1}{2} c_{3}-\frac{1}{2} a_{0}+i a_{1}, \quad \alpha_{1(0)}^{\prime}=-\frac{1}{2} c_{3}-\frac{1}{2} a_{0}-i a_{1}$,

$\alpha_{1(1)}=c_{1}+c_{3}-\frac{9}{8}+i\left(-2 a_{1}+a_{2}+3 a_{5}\right)$,

$\alpha_{1(1)}^{\prime}=c_{1}+c_{3}-\frac{9}{8}-i\left(-2 a_{1}+a_{2}+3 a_{5}\right)$,

$\alpha_{1(k \geq 2)}=\frac{(-2)^{k-1}}{k !}\left[\frac{1}{2} c_{1}+c_{3}-\frac{1}{2}-i\left(2 a_{1}-a_{2}-4 a_{5}\right)\right.$

$$
\left.+i(k-2) a_{5}\right] \text {, }
$$

$\alpha_{1(k \geq 2)}^{\prime}=\frac{(-2)^{k-1}}{k !}\left[\frac{1}{2} c_{1}+c_{3}-\frac{1}{2}+i\left(2 a_{1}-a_{2}-4 a_{5}\right)\right.$

$$
\left.-i(k-2) a_{5}\right] \text {, }
$$

$\alpha_{2(0)}=\frac{1}{2} c_{1}-c_{3}-\frac{3}{4}+i a_{2}, \quad \alpha_{2(0)}^{\prime}=\frac{1}{2} c_{1}-c_{3}-\frac{3}{4}-i a_{2}$,

$\alpha_{2(1)}=2 c_{1}+2 c_{3}-\frac{9}{4}+6 i a_{5}, \quad \alpha_{2(1)}^{\prime}=2 c_{1}+2 c_{3}$

$$
-\frac{9}{4}-6 i a_{5}
$$

$\alpha_{2(k \geq 2)}=\frac{(-2)^{k-1}}{k !}\left[c_{1}+2 c_{3}-1+2 i(k+2) a_{5}\right]$,

$\alpha_{2(k \geq 2)}^{\prime}=\frac{(-2)^{k-1}}{k !}\left[c_{1}+2 c_{3}-1-2 i(k+2) a_{5}\right]$,

$\alpha_{3(0)}=-\frac{3}{2} c_{1}^{2}+\frac{7}{2} c_{1}-2 c_{1} c_{3}+s \frac{1}{2} c_{2}^{2}-2 c_{3}^{2}+2 c_{3}-\frac{11}{8}+i a_{3}$, $\alpha_{3(0)}^{\prime}=-\frac{3}{2} c_{1}^{2}+\frac{7}{2} c_{1}-2 c_{1} c_{3}+\frac{1}{2} c_{2}^{2}-2 c_{3}^{2}$

$$
+2 c_{3}-\frac{11}{8}-i a_{3} \text {, }
$$

$\alpha_{3(k \geq 1)}=\frac{(-2)^{k}}{k !} i a_{3}, \quad \alpha_{3(k \geq 1)}^{\prime}=-\frac{(-2)^{k}}{k !} i a_{3}$,

$\alpha_{4(0)}=\frac{9}{8}-c_{1}-c_{3}-i\left(a_{2}+3 a_{5}\right)$,

$\alpha_{4(0)}^{\prime}=\frac{9}{8}-c_{1}-c_{3}+i\left(a_{2}+3 a_{5}\right)$,

$\alpha_{4(1)}=\frac{1}{2} c_{1}+2 c_{3}-\frac{3}{8}+3 i a_{5}$,

$\alpha_{4(1)}^{\prime}=\frac{1}{2} c_{1}+2 c_{3}-\frac{3}{8}-3 i a_{5}$,

$\alpha_{4(k \geq 2)}=\frac{(-2)^{k-1}}{k !}\left[c_{1}+2 c_{3}-1+i(k+2) a_{5}\right]$,

$\alpha_{4(k \geq 2)}^{\prime}=\frac{(-2)^{k-1}}{k !}\left[c_{1}+2 c_{3}-1-i(k+2) a_{5}\right]$,

$\alpha_{5(0)}=\frac{5}{8}-\frac{1}{2} c_{1}+i a_{5}, \quad \alpha_{5(0)}^{\prime}=\frac{5}{8}-\frac{1}{2} c_{1}-i a_{5}$,

$\alpha_{5(k \geq 1)}=\frac{(-2)^{k}}{k !} i a_{5}, \quad \alpha_{5(k \geq 1)}^{\prime}=-\frac{(-2)^{k}}{k !} i a_{5}$,

$\beta_{1(0)}=b_{0}+i b_{1}, \quad \beta_{1(0)}^{\prime}=b_{0}-i b_{1}$,

$\beta_{1(1)}=\frac{5}{2} c_{1}^{2}+5 c_{3} c_{1}-5 c_{1}+\frac{1}{2} c_{2}^{2}+2 c_{3}^{2}-4 c_{3}+c_{2} c_{4}+\frac{19}{8}$,

$+3 i c_{4} c_{1}+4 i c_{2} c_{1}-3 i c_{2}$

$+3 i c_{2} c_{3}-3 i c_{4}+3 i a_{2}+i a_{3}$

$+6 i a_{5}-4 i b_{1}-2 i b_{2}$,

$\beta_{1(1)}^{\prime}=\beta_{1(1)}^{*}$,

$\beta_{1(2)}=-\frac{5}{2} c_{1}^{2}-5 c_{3} c_{1}+4 c_{1}-\frac{1}{2} c_{2}^{2}+3 c_{3}-c_{2} c_{4}-\frac{3}{2}$

$-4 i c_{2} c_{1}-3 i c_{4} c_{1}+3 i c_{2}-3 i c_{2} c_{3}+3 i c_{4}$

$-3 i a_{2}-i a_{3}-6 i a_{5}+6 i b_{1}+3 i b_{2}$,

$\beta_{1(2)}^{\prime}=\beta_{1(2)}^{*}$,

$\beta_{1((k \geq 2)}=\frac{(-2)^{k-2}}{k !}\left[2 \beta_{1(2)}+(k-2)\left[\xi_{0(0)} \gamma_{0(1)}+\gamma_{1(1)}\right]\right]$,

$\beta_{1((k \geq 2)}^{\prime}=\beta_{1((k \geq 2)}^{*}$,

$\beta_{2(0)}=-\frac{7}{8}+c_{1}+c_{3}-2 c_{3}^{2}+i b_{2}$,

$\beta_{2(0)}^{\prime}=\frac{7}{8}-c_{1}-c_{3}+2 c_{3}^{2}+i b_{2}$,

$\beta_{2(1)}=-\frac{19}{4}-6 c_{1}^{2}+11 c_{1}-12 c_{1} c_{3}+8 c_{3}$

$-4 c_{3}^{2}-2 i c_{1} c_{2}-2 i a_{3}$,

$\beta_{2(1)}^{\prime}=\frac{19}{4}+6 c_{1}^{2}-11 c_{1}+12 c_{1} c_{3}-8 c_{3}$

$+4 c_{3}^{2}-2 i c_{1} c_{2}-2 i a_{3}$,

$\beta_{2(2)}=3\left(1-2 c_{1}\right)\left(1-c_{1}-2 c_{3}\right)+2 i c_{1} c_{2}+2 i a_{3}$,

$\beta_{2(2)}^{\prime}=-3\left(1-2 c_{1}\right)\left(1-c_{1}-2 c_{3}\right)+2 i c_{1} c_{2}+2 i a_{3}$,

$\beta_{2((k \geq 2)}=-\frac{(-2)^{k-1}}{k !}\left[(k+1)\left(1-2 c_{1}\right)\left(1-c_{1}-2 c_{3}\right)\right.$

$\left.+2 i c_{1} c_{2}+2 i a_{3}\right]$,

$\beta_{2(k \geq 2)}^{\prime}=\frac{(-2)^{k-1}}{k !}\left[(k+1)\left(1-2 c_{1}\right)\left(1-c_{1}-2 c_{3}\right)\right.$

$\left.-2 i c_{1} c_{2}-2 i a_{3}\right]$, 


$$
\begin{aligned}
\gamma_{1(0)}= & \frac{7}{8}-c_{1}-c_{3}+2 c_{3}^{2}-2 i b_{1}-i b_{2}, \\
\gamma_{1(0)}^{\prime}= & \frac{7}{8}-c_{1}-c_{3}+2 c_{3}^{2}+2 i b_{1}+i b_{2}, \\
\gamma_{1(1)}= & \left(1-2 c_{1}\right)\left(1-c_{1}-2 c_{3}\right) \\
& -2 i c_{2} c_{1}-2 i c_{4} c_{1}+2 i c_{2}-2 i c_{2} c_{3} \\
& +2 i c_{4}-2 i a_{2}-4 i a_{5}+4 i b_{1}+2 i b_{2}, \\
\gamma_{1(1)}^{\prime}= & \gamma_{1(1)}^{*}, \quad \gamma_{1(k \geq 1)}=\frac{(-2)^{k-1}}{k !} \gamma_{1(1)}, \\
\gamma_{1(k \geq 1)}^{\prime}= & \frac{(-2)^{k-1}}{k !} \gamma_{1(1)}^{*}, \\
\gamma_{2(0)}= & -\gamma_{2(0)}^{\prime}=\frac{7}{4}-2 c_{1}-2 c_{3}+4 c_{3}^{2}, \\
\gamma_{2(k \geq 1)}= & -\gamma_{2(k \geq 1)}^{\prime}=-\frac{(-2)^{k}}{k !}\left(1-2 c_{1}\right)\left(1-c_{1}-2 c_{3}\right)
\end{aligned}
$$

Here the independent constants are $c_{1}, c_{2}, c_{4}, a_{0}, a_{1}, a_{2}, a_{3}$, $a_{5}, b_{0}, b_{1}, b_{2}$, and $c_{3}$ obey the equation

$6 c_{1}^{2}+12 c_{3} c_{1}-9 c_{1}+4 c_{3}^{2}-6 c_{3}+3=0$,

and $c_{3}$ has the form $c_{3}=\frac{1}{4}\left(3-6 c_{1} \pm \sqrt{3} \sqrt{4 c_{1}^{2}-1}\right)$.

\section{References}

1. M.A. Vasiliev, Fortsch. Phys. 52, 702 (2004)

2. D. Sorokin, Introduction to the classical theory of higher spins. AIP Conf. Proc. 767, 172 (2005). arXiv:hep-th/0405069

3. X. Bekaert, S. Cnockaert, C. Iazeolla, M.A. Vasiliev, Nonlinear higher spin theories in various dimensions (2009). arXiv:hep-th/0503128

4. A. Fotopoulos, M. Tsulaia, Int. J. Mod. Phys. A 24, 1 (2009)

5. X. Bekaert, N. Boulanger, P. Sundell, Rev. Mod. Phys. 84, 987 (2012)

6. A. Sagnotti, J. Phys. A 46, 214006 (2013)

7. V.E. Didenko, E.D. Skvortsov, Elements of Vasiliev theory (2015), arXiv:1401.2975 [hep-th]

8. M.A. Vasiliev, Lect. Notes Phys. 892, 227 (2015)

9. E.S. Fradkin, M.A. Vasiliev, Phys. Lett. B 189, 89 (1987)

10. E.S. Fradkin, M.A. Vasiliev, Nucl. Phys. B 291(1987), 141 (1987)

11. R.R. Metsaev, Nucl. Phys. B 759, 147 (2006)

12. I.L. Buchbinder, A. Fotopoulos, A.C. Petkou, M. Tsulaia, Phys. Rev. D 74, 105018 (2006)

13. A. Fotopoulos, N. Irges, A.C. Petkou, M. Tsulaia, JHEP 0710, 021 (2007)

14. M. Porrati, R. Rahman, Nucl. Phys. B 814, 370-404 (2009)

15. X. Bekaert, N. Boulanger, S. Leclercq, J. Phys. A 43, 185401 (2010)

16. R. Manvelyan, K. Mkrtchyan, W. Ruhl, Nucl. Phys. B 836, 204 (2010)

17. R. Manvelyan, K. Mkrtchyan, W. Ruehl, Phys. Lett. B 696, 410 (2011)

18. N. Boulanger, E.D. Skvortsov, JHEP 1109, 063 (2011)

19. N. Boulanger, E.D. Skvortsov, Y.M. Zinoviev, J. Phys. A 44, 415403 (2011)

20. R.R. Metsaev, Nucl. Phys. B 859, 13 (2012)

21. M. Taronna, Higher-spin interactions: three-point functions and beyond (2013). arXiv:1209.5755 [hep-th]

22. N. Boulanger, D. Ponomarev, E.D. Skvortsov, JHEP 1305, 008 (2013)

23. E. Joung, L. Lopez, M. Taronna, J. Phys. A 46, 214020 (2013)

24. E. Joung, L. Lopez, M. Taronna, JHEP 1301, 168 (2013)

25. M. Henneaux, R. Rahman, Phys. Rev. D 88, 064013 (2013)
26. Y.M. Zinoviev, Nucl. Phys. B 872, 21 (2013)

27. Y.M. Zinoviev, Nucl. Phys. B 886, 712 (2014)

28. S.M. Klishevich, Y.M. Zinovev, Phys. Atom. Nucl. 61, 1527 (1998)

29. S.M. Klishevich, Y.M. Zinovev, Yad. Fiz. 61, 1638 (1998)

30. S.M. Klishevich, Class. Quant. Grav. 16, 2915 (1999)

31. M. Porrati, R. Rahman, A. Sagnotti, Nucl. Phys. B 846, 250 (2011)

32. I.L. Buchbinder, T.V. Snegirev, YuM Zinoviev, Nucl. Phys. B 864, 694 (2012)

33. I.L. Buchbinder, P. Dempster, M. Tsulaia, Nucl. Phys. B 877, 260 (2013)

34. I. Cortese, R. Rahman, M. Sivakumar, Nucl. Phys. B 879, 143 (2014)

35. M. Kulaxizi, R. Rahman, JHEP 1410, 193 (2014)

36. S.M. Klishevich, Int. J. Mod. Phys. A 15, 535 (2000)

37. M. Taronna, JHEP 1204, 029 (2012)

38. P. Dempster, M. Tsulaia, Nucl. Phys. B 865, 353 (2012)

39. G. Velo, D. Zwanziger, Phys. Rev. 186, 1337 (1969)

40. G. Velo, D. Zwanziger, Phys. Rev. 188, 2218 (1969)

41. G. Velo, Nucl. Phys. B 43, 389 (1972)

42. I.L. Buchbinder, V.A. Krykhtin, P.M. Lavrov, Mod Phys. Lett. A 26, 1183-1196 (2011)

43. I.L. Buchbinder, V.A. Krykhtin, A. Pashnev, Nucl. Phys. B 711, 367 (2005)

44. I.L. Buchbinder, V.A. Krykhtin, Nucl. Phys. B 727, 537 (2005)

45. I.L. Buchbinder, V.A. Krykhtin, L.L. Ryskina, H. Takata, Phys. Lett. B 641, 386 (2006)

46. I.L. Buchbinder, V.A. Krykhtin, H. Takata, Phys. Lett. B 656, 253 (2007)

47. I.L. Buchbinder, V.A. Krykhtin, P.M. Lavrov, Nucl. Phys. B 762, 344 (2007)

48. I.L. Buchbinder, V.A. Krykhtin, A.A. Reshetnyak, Nucl. Phys. B 787, 211 (2007)

49. I.L. Buchbinder, V.A. Krykhtin, L.L. Ryskina, Mod. Phys. Lett. A 24, 401 (2009)

50. I.L. Buchbinder, V.A. Krykhtin, L.L. Ryskina, Nucl. Phys. B 819, 453 (2009)

51. I.L. Buchbinder, V.A. Krykhtin, P.M. Lavrov, Phys. Lett. B 685 , 208 (2010)

52. I.L. Buchbinder, V.A. Krykhtin, Mod. Phys. Lett. A 25, 1667 (2010)

53. I.L. Buchbinder, A.V. Galajinsky, V.A. Krykhtin, Nucl. Phys. B 779, 155 (2007)

54. I.L. Buchbinder, A.V. Galajinsky, JHEP 0811, 081 (2008)

55. A.I. Pashnev, Theor. Math. Phys. 78, 272 (1989)

56. A.I. Pashnev, Teor. Mat. Fiz. 78, 384 (1989)

57. D. Francia, A. Sagnotti, Class. Quant. Grav. 20, S473 (2003)

58. D. Francia, A. Sagnotti, Comment. Phys. Math. Soc. Sci. Fenn. 166, 165 (2004)

59. D. Francia, A. Sagnotti, PoS JHW 2003, 005 (2003). arXiv:hep-th/0212185

60. A. Sagnotti, M. Tsulaia, Nucl. Phys. B 682, 83 (2004)

61. D. Francia, Phys. Lett. B 690, 90 (2010)

62. A. Campoleoni, D. Francia, JHEP 1303, 168 (2013)

63. D.P. Sorokin, M.A. Vasiliev, Nucl. Phys. B 809, 110 (2009)

64. X. Bekaert, N. Boulanger, D. Francia, Mixed-symmetry multiplets and higher-spin curvatures (1974). arXiv: 1501.02462 [hep-th]

65. L.P.S. Singh, C.R. Hagen, Phys. Rev. D 9, 898 (1974)

66. I.L. Buchbinder, V.A. Krykhtin, M. Tsulaia, Nucl. Phys. B 896, 1 (2015)

67. Yu.M. Zinoviev, Nucl. Phys. B 785, 98-114 (2007)

68. I. Florakis, D. Sorokin, M. Tsulaia, JHEP 1407, 105 (2014)

69. I. Florakis, D. Sorokin, M. Tsulaia, Nucl. Phys. B 890, 279 (2014)

70. X. Bekaert, J. Erdmenger, D. Ponomarev, C. Sleight, JHEP 1503, $170(2015)$

71. R.R. Metsaev, Phys. Rev. D 81, 106002 (2010). arXiv:0907.4678 [hep-th] 
72. R.R. Metsaev, CFT adapted approach to massless fermionic fields,

74. R.R. Metsaev, JHEP 1501, 077 (2015) AdS/CFT, and fermionic conformal fields. arXiv:1311.7350 [hep-

75. T. Nutma, M. Taronna, JHEP 1406, 066 (2014) th]

73. R.R. Metsaev, Theor. Math. Phys. 181(3), 1548 (2014) 